\title{
Prospects for Beyond Standard Model physics searches at Hyper-Kamiokande and T2K
}

\author{
R. Phillip Litchfield* \\ University of Glasgow \\ E-mail: Phillip.Litchfieldeglasgow.ac.uk
}

\begin{abstract}
The Super-Kamiokande and T2K experiments have substantially developed our understanding of oscillations by observing the subdominant electron-neutrino appearance channel. The nextgeneration Hyper-Kamiokande experiment will build on this with much higher statistics, enabling precision tests of the Standard PMNS picture. In the baseline design of two tanks at Kamioka, a detailed investigation of oscillations can be made in the vicinity of the first oscillation maximum, and at short baselines using the near detector. More interesting tests can be performed if the second tank is located in Korea, as this gives access to an $L / E$ regime that is three times higher than any previous long-baseline experiment. As well as benefits to the PMNS measurements, a new experimental regime makes it possible to resolve BSM models which are statistically degenerate on a single baseline. This talk focuses on three models: Lorentz violation with neutrinos; sterile neutrinos; and non-standard interactions.
\end{abstract}

The 21st international workshop on neutrinos from accelerators (NuFact2019)

August 26 - August 31, 2019

Daegu, Korea

\footnotetext{
* Speaker.

${ }^{\dagger}$ On behalf of the Hyper-Kamiokande collaboration
} 


\section{Introduction and scope}

The topic I was asked to speak about is Beyond Standard Model physics at Hyper-Kamiokande (Hyper-K). Because that is a broad topic, it was necessary to narrow it down to fit into the available time. Since this is NuFact, the focus is on neutrino physics using accelerator sources. So, regretfully, this talk does not cover some important parts of the Hyper-K physics programme: natural neutrino sources, such as solar or cosmogenic neutrinos; other astrophysical topics, such as indirect dark matter searches; searches for non-neutrino components of the beam; and, notably, nucleon decay. The three topics that will be presented are: Lorentz violation; light sterile neutrinos; and non-standard interactions.

\section{(Very) brief introduction to Hyper-K and the JPARC beam}

The existing T2K experiment makes use of a muon neutrino beam originating at from JPARC, on Japan's east coast, and directed almost exactly due west, towards Super-Kamiokande detector, $295 \mathrm{~km}$ away. In the baseline Hyper-K design the experiment is upgraded with a more powerful beam and two new tanks, for a fiducial mass around an order of magnitude larger. This baseline design is supplemented with two more detector sites. The first is an Intermediate Water-Chernekov Detector (IWCD) at $2 \mathrm{~km}$ from the beam source, which will be movable and capable of scanning a range of energy spectra by making use of the off-axis effect. The second modification involves siting one of the large tanks in South Korea, at a little over $1000 \mathrm{~km}$ from the JPARC site. This detector in this configuration is termed the Korea Neutrino Observatory, while the overall beamlineplus-detectors configuration is referred to here as T2HK/Korea. This would allow the experiment to investigate an $L / E$ regime that is around three times larger than any existing accelerator neutrino experiment, around the second disappearance maximum. General details of the T2K [1] and HyperKamiokande [2] experiments, and the KNO extension [3] were presented in the plenary sessions. Details on Hyper-K performance are based on published design reports [4, 5], unless specified.

\section{Lorentz violation}

The Standard Model Extension (SME) of Lorentz violation [6, 7] allows flavour-violating modifications to the neutrino propagation Hamiltonian, expressible a perturbation of the usual PMNS model. In general, the first few expansion terms look like:

$$
P\left(v_{a} \rightarrow v_{b}\right) \sim\left|S_{a b}^{(0)}\right|^{2}+\underbrace{2 \mathfrak{R e}\left[\left(S_{a b}^{(0)}\right)^{*} S_{a b}^{(1)}\right]}_{\text {LBL }}+2 \mathfrak{R e}\left[\left(S_{a b}^{(0)}\right)^{*} S_{a b}^{(2)}\right]+\underbrace{\left.\mid S_{a b}^{(1)}\right]}_{\mathrm{SBL}}+\ldots
$$

where the index in brackets is the order of the perturbation, and $S_{a b}^{(0)}$ is the unperturbed PMNS propagation. The two most interesting terms in the series are marked. The first (Long Baseline) term describes the leading order interference with regular PMNS oscillations, and is dominant if regular oscillations are large. The second (Short Baseline) term is higher order in the perturbation, but is does not involve the oscillation so can give rise to effects even if standard oscillations have not developed. 
The fact that both these terms exists means it is possible to look for Lorentz violation in both Near and Far detectors. The 'smoking gun' for the SME arises because the Lorentz violating parameters are fixed 4-vectors or tensors. This means the perturbation would show up with a sidereal variation (period $T_{\oplus}=23^{h} 56^{m} 4.1^{s}$ or harmonics thereof) as the earth rotates against the fixed stars, and the angle between the neutrino momentum and the SME parameters changes. The resulting changes to the oscillation probability also increase with baseline, and the effect of tensorlike terms also increases with neutrino energy.

To date, T2K has published a statistically-limited null analysis of sidereal variation in the INGRID near detector data, [8] looking for a variation with signature:

$$
P^{\mathrm{SBL}}\left(v_{a} \rightarrow v_{b}\right)=L^{2}\left|\mathcal{C}_{a b}+\mathcal{A}_{a b} \sin \left(\omega_{\oplus} t+\phi\right)+\mathcal{B}_{a b} \sin \left(2 \omega_{\oplus} t+\phi\right)\right|^{2}
$$

and results in constrains of $\sim 3 \times 10^{-20}$ (95\%) on the oscillating components. A future analysis using the IWCD would benefit from the longer baseline and $L^{2}$ dependence resulting in an effect size that is around two order of magnitude larger than for ND280

In addition Hyper-K (\& T2K) can look for the sidereal variation of the PMNS-LV interference term which has a linear $L$ dependence, and is enhanced because the PMNS oscillation is large:

$$
\Delta P^{\mathrm{LBL}}\left(v_{a} \rightarrow v_{b}\right)=2 L\left(\left(P_{\mathcal{C}}\right)_{a b}+\left(P_{\mathcal{A}}\right)_{a b} \sin \left(\omega_{\oplus} t+\phi\right)+\left(P_{\mathcal{B}}\right)_{a b} \sin \left(2 \omega_{\oplus} t+\phi\right)\right) .
$$

In this case the effect size is largest a the Korean detector as it grows with baseline. In addition the higher energy enhances the tensor parameter contribution relative to the vector parameters.

\section{Sterile neutrinos}

Arguably the simplest way to extend the PMNS model is to increase the number of neutral leptons. If there are new lepton(s) with mass(es) $m_{4} \gtrsim 2 m_{e}$ then they may be observable through decay to normal neutrinos, and are termed Heavy Neutral Leptons. For this talk we focus on the case where $m_{4} \lesssim m_{e}$. In this case, the leptons can take part in oscillations and reveal themselves through precision measurements. Such neutrinos are termed 'sterile', since LEP $Z^{0}$ data excludes a fourth light neutrino carrying weak charge.

Most experiments looking for sterile neutrinos look for evidence of a new mass state, via oscillations between active neutrinos at a large new mass splitting $\Delta m_{41}^{2}$, and hence at short baselines. An alternative approach is to look for the fourth flavour state, which can have no weak interactions. If it exists it would reduce the observed rate of Neutral Current events at the normal atmosphericscale L/E oscillation scale. T2K is - and Hyper-K will be - capable of both approaches.

T2K has done a short baseline analysis of ND280 data [9] looking for depletion of the $v_{e}$ component, but the analysis was statistically limited and is inconclusive for most of the parameter space considered interesting. More recently a joint analysis of Super-K and ND280 data [10] was able to set competitive limits for small values of $\Delta m_{41}^{2}$. Both of these analyses could be updated for Hyper-K.

The most interesting prospect for sterile neutrino analyses uses the IWCD. With the ND280 to constrain the flux, this detector can be used for a precision measurement of the $v_{\mu} \rightarrow v_{e}$ oscillation channel in a regime very similar to the LSND/MiniBooNE signals. Sensitivity studies [11] show 
that if there is no signal such an analysis could exclude the entire LSND 90\% allowed region at $90 \%$ C.L. and the best fit point at around $5 \sigma$. The fact that the IWCD can move to different off-axis angles to change the neutrino energy spectrum can also be used to constrain backgrounds in a way that is not possible for a static detector configuration.

\section{Non-standard interactions}

A different extension of the PMNS model relates to the matter effect. The generalised matter effect corresponds to a flavour-dependant contribution to the neutrino propagation Hamiltonian:

$$
\mathcal{H}=\frac{1}{2 E} U_{\mathrm{PMNS}}\left(\begin{array}{ccc}
0 & 0 & 0 \\
0 & \Delta m_{21}^{2} & 0 \\
0 & 0 & \Delta m_{31}^{2}
\end{array}\right) U_{\mathrm{PMNS}}^{\dagger}+V_{e}\left(\begin{array}{ccc}
1+\varepsilon_{e e} & \varepsilon_{e \mu} & \varepsilon_{e \tau} \\
\varepsilon_{e \mu}^{*} & \varepsilon_{\mu \mu} & \varepsilon_{\mu \tau} \\
\varepsilon_{e \tau}^{*} & \varepsilon_{\mu \tau}^{*} & \varepsilon_{\tau \tau}
\end{array}\right)
$$

In terrestrial long baseline experiments these arise from coherent forward scattering of the neutrinos from the electrons and quarks of the Earth's crust. The standard model matter effect includes two Weak components: a flavour-independent NC contribution, which (like the absolute mass scale) is not observable, and a $v_{e}$-specific charged current term, which is equal to $V_{e}=\sqrt{2} G_{\mathrm{F}} \rho$ and contributes only to the top left $e e$ element. Models termed Non-Standard Interactions (NSI) have additional $\varepsilon_{\alpha \beta}$ terms in the propagator that represent soft contributions from new physics, and can arise in (e.g.) models of neutrino mass generation. The magnitude of these parameters are typically only constrained to the same level as the Weak interaction: $\mathcal{O}(0.1) \sim \mathcal{O}(1)$, and Hyper-K can equal or improve on current global limits [12] on $\varepsilon_{\tau \tau}, \varepsilon_{e e}, \varepsilon_{e \mu}$ and $\varepsilon_{e \tau}$. The T2HK/Korea configuration is particularly sensitive as it benefits from a higher beam energy, and a longer baseline so there is more time for discrepancies from PMNS mixing to develop, and can improve current limits on $\varepsilon_{e \mu}$ and $\varepsilon_{e e}$, down to $\gtrsim 0.1$ and $\gtrsim 0.5$ respectively [13].

Though the discovery of NSI would be of great interest, there is a 'conjugate' problem. The NSI parameter space also includes complex phases of the off-diagonal elements, which allow for parameter combinations that are (statistically) degenerate with the PMNS model. In this case there would be no conclusive evidence of new physics, but our deductions about the PMNS parameter $\delta_{\mathrm{CP}}$, would also be incorrect. An analysis of the degeneracy [13] shows that—of current and nextgeneration experiments—only the T2HK/Korea configuration is free from it, and therefore still capable of a precise measurement of $\delta_{\mathrm{CP}}$.

\section{Summary}

The three models described are not the only BSM physics covered by Hyper-K and T2K. As well as the non-neutrino BSM models, modifications to the $L$ and $E$ dependence from neutrino decay or decoherence can be checked, particularly with the T2HK/Korea configuration. However such models have not been analysed by T2K, nor studied for Hyper-K. The models for which studies have been done either as T2K analyses or specifically for Hyper-K are summarised in Table 1. Considering that the amount of CPV violation in the target $v_{\mu} \rightarrow v_{e}$ oscillation channel may be large, searches for BSM physics will be an important part of the experimental program, as the experiments may well transition from a simple goal of discovery to precision tests of the PMNS 
model. Any discovery of new physics may also point toward a model of neutrino mass, something that standard oscillation analyses cannot directly constrain.

Table 1: Summary of BSM models for which Hyper-K beam studies are available, or are feasible based on extrapolation from published T2K analyses.

\begin{tabular}{|c|c|c|c|}
\hline & Lorentz violation & Sterile neutrinos & $\begin{array}{l}\text { Non-standard } \\
\text { interactions }\end{array}$ \\
\hline Studied by T2K? & $\checkmark$ & $\checkmark$ & \\
\hline Systematic limit? & Not yet & Same as $3 v$ & \\
\hline \multirow[t]{2}{*}{ Hyper-K design study? } & & $\checkmark(\mathrm{SBL})$ & $\checkmark$ \\
\hline & Larger effect at IWCD & $\begin{array}{l}\text { IWCD a near-perfect } \\
\text { test of LSND / MB }\end{array}$ & $\begin{array}{l}\text { Order-of-magnitude } \\
\text { improvement }\end{array}$ \\
\hline \multirow[t]{2}{*}{ Prospects and notes } & LBL analysis & Unified SBL and LBL & \\
\hline & $\begin{array}{l}4 \text { baselines can break } \\
\text { degeneracies }\end{array}$ & $\begin{array}{l}\text { Not yet studied with } \\
\text { (LBL) Hyper-K }\end{array}$ & $\begin{array}{l}\text { Only } \mathrm{T} 2 \mathrm{HK} / \text { Korea has } \\
\text { robust } \delta_{\mathrm{CP}} \text { result }\end{array}$ \\
\hline
\end{tabular}

\section{References}

[1] F. Bench, "The T2K Experiment: Current Status and Results", also presented at NuFact2019

[2] T. Kobayashi, "Hyper-Kamiokande", also presented at NuFact2019

[3] I. Yu, "KNO Report", also presented at NuFact2019

[4] K. Abe et al. [Hyper-Kamiokande Collaboration], arXiv:1805.04163

[5] K. Abe et al. [Hyper-Kamiokande Collaboration], PTEP 2018, no. 6, $063 \mathrm{C} 01$ (2018)

[6] V. A. Kostelecky and M. Mewes, Phys. Rev. D70, 076002 (2004)

[7] J. S. Diaz, V. A. Kostelecky and M. Mewes, Phys. Rev. D80, 076007 (2009)

[8] K. Abe et al. [T2K collaboration], Phys. Rev. D95, no. 11, 111101 (2017)

[9] K. Abe et al. [T2K collaboration], Phys. Rev. D91 051102 (2015)

[10] K. Abe et al. [T2K collaboration], Phys. Rev. D99 071103 (2019)

[11] S. Bhadra et al. [NuPRISIM collaboration] "Proposal for the NuPRISM Experiment in the J-PARC Neutrino Beamline" Submission to JPARC PAC

[12] K. N. Deepthi, S. Goswami and N. Nath, Nucl. Phys. B 936, 91 (2018)

[13] J. Liao, D. Marfatia and K. Whisnant, JHEP 1701, 071 (2017) 